\title{
A Group Recommender for Investment in Microgrid Renewable Energy Sources
}

\author{
Hanan Mengash \\ Princess Nourah bint Abdulrahman University \\ Riyadh, Saudi Arabia \\ hamengash@pnu.edu.sa
}

\author{
Alexander Brodsky \\ George Mason University \\ Fairfax, VA 22030, USA \\ brodsky@gmu.edu
}

\begin{abstract}
Integration of renewable energy sources such as photovoltaic arrays and wind turbines into electric power microgrids can significantly reduce greenhouse gas (GHG) emissions. However, deciding on investment in microgrid renewable energy sources is a complex problem due to (1) the space of alternatives which is exponential in a number of components; (2) the complex interactions between old and new equipment in every time interval over an investment time horizon; (3) the multiple criteria that should be considered such as net present value, GHG emissions, and system reliability; and (4) dealing with a group of decision makers with diverse priorities. In this paper, we propose and report on the development of a Power Microgrid Operation and Investment Recommender (PMOIR) to guide a group of decision makers toward investment decisions on microgrid renewable energy sources. This is done under the assumption of optimal operational control over the investment time horizon. PMOIR uses a framework of extracting user preferences, estimating the group utility, optimizing and diversifying a small number of recommended alternatives, and voting. To support optimization, we mathematically model different power components and formalize the overall optimization problem, which is implemented using a mixed integer linear programming model. We also conduct an experimental study to demonstrating PMOIR feasibility, in terms of computational time, to be applied on microgrids involving 200 power components, over a five-year time horizon, with around 8 million binary variables.
\end{abstract}

\section{Introduction}

A microgrid is an integrated system of energy resources together with a sophisticated decision system for controlling them, such as the power microgrid of a university campus, an industrial facility, or a building complex (see Figure1). Recently, microgrids involving renewable energy sources like solar and wind energy are considered as a high-quality and reliable source of electricity, while contributing to reduction of greenhouse gas (GHG) emissions. However, deciding on investment in microgrid renewable energy sources is a complex problem due to (1) the space of alternatives which is exponential in a number of components; (2) the complex interactions between old and new equipment in every time interval over an investment time horizon; (3) the multiple criteria that should be considered such as net present value (NPV), GHG emissions, and system reliability, which all will have different values for each different combination of the investment components; and (4) dealing with a group of decision makers with diverse priorities [5]. Group recommenders are considerably more complex than individual user recommenders. One reason for this complexity is the need to effectively aggregate users' preferences in a way that maximizes the group's satisfaction, fairness, and user-friendliness.

For a complex problem like this, a question arises: How to guide a group of decision makers, with different or even conflicting priorities, toward investment decisions on microgrid renewable energy sources having multiple criteria, e.g., NPV, GHG emissions, and demand satisfaction ratio. This is exactly the focus of this paper.

Most of the existing work in microgrid investment (e.g., [19]) considered either renewable energy investment at a long time horizon (years) or demand response optimization at a short time horizon (days or hours) (e.g., [18]). However, optimal investment decisions should be done with an assumption that the acquired resources operate optimally, and thus the two optimization should be considered simultaneously. 
Recently, some work considered optimization of investment and operation (e.g., [3, 17]), however, they focused only on a single criterion (cost), and on specific power components. Also, their systems are not designed to support group of decision makers, with conflicting priorities.

For existing recommender systems, there has been extensive work that mostly focused on single-users rather than groups (e.g., [16]). Recently, researchers have proposed group recommenders in different domains and applications that used different strategies to aggregate individual preferences into a group model [9]. However, most of these group recommender systems were designed for atomic products or services rather than for automatically constructed packages of product and services. In addition, the majority of recommender systems rely on a single ranking or utility score, whereas in many applications there are multiple criteria that need to be taken into account.

Recently, there has been some research on package recommendations [6]. However, they do not consider and/or use dynamic preference learning and decision optimization. Work [4] provides package recommendations based on dynamic preference learning and decision optimization. However, it focused on individuals rather than groups.

More recently, it has been proposed in [10-12] a group composite alternatives recommender (GCAR) framework that addresses the outlined limitations, and provides a diverse set of group package recommendations based on multi-criteria decision optimization. However, this framework is a generic one and has not been applied on a realistic complex problem, like the microgrid renewable energy sources investment.

Developing a Power Microgrid Operation and Investment Recommender (PMOIR) using GCAR Framework on a real life complex problem of investing in renewable energy sources is the focus of this paper. More specifically, the contributions of this paper are: First, we propose and report on the development of a Power Microgrid Operation and Investment Recommender (PMOIR) to guide a group of decision makers toward investment decisions on microgrid renewable energy sources. This is done under the assumption of optimal operational control over the investment time horizon. PMOIR uses a framework of extracting user preferences, estimating the group utility, optimizing and diversifying a small number of recommended alternatives, and voting.

Second, to support optimization, we mathematically model different power components and formalize the overall optimization problem, which is implemented as a mixed-integer linear programming
(MILP) model using IBM Optimization Programming Language (OPL) and CPLEX Studio.

Finally, we conduct an experimental study demonstrating the PMOIR feasibility, in terms of computational time, to be applied on microgrids involving 200 power components, over a five-year time horizon, with around 8 million binary variables.

This paper is organized as follows: Section 2 details the related work and its limitations. Section 3 gives a high level description of PMOIR. Section 4 gives a high-level description of the GCAR framework. Section 5 describes the optimization formalization. Section 6 describes the power component modeling. Section 7 presents the experimental study. Section 8 is the conclusion and avenues for future work.

\section{Related Work}

In recent years, there are many works on power microgrid planning, renewable energy investment, and demand response. For example, work [19] considered investment strategies on renewable energy sources, but without considering the power microgrid operation. Work [18] focused on the study of optimal demand response through optimization models, but without considering renewable energy investment. More recently, joint optimization of investment and operation has been considered in some works (e.g., [3, 17]), however they focused only on a single criterion (cost), and on specific power components. Also, their systems are not designed to support group of decision makers, with conflicting priorities.

For existing group recommenders, researchers have proposed systems in different domains and applications that used different strategies to aggregate individual preferences into a group model. Common examples of group recommender systems include: recommending TV programs and movies, finding songs to play at a shared public space, or finding tourist attraction for a group of tourists [9]. However, most of the existing group recommender systems were not designed for automatically constructed packages of products and services, which makes the recommendation space very large, or even infinite, and implicitly, rather than explicitly, defined.

Recently, there has been some research on packages recommendations [6], however, they do not consider and/or use dynamic preference learning and decision optimization. Work [4] support packages of product and service definitions, and provide recommendations based on dynamic preference learning and decision optimization. However, it is a 
recommender system for individuals rather than groups.

In addition, the majority of recommender systems rely on a single ranking or utility score, whereas in many applications there are multiple criteria that need to be taken into account. Recently, few existing multicriteria recommender systems have roots in multicriteria optimization techniques (e.g., [7]); however, these systems focus on atomic (single) products, rather than composite products, and on individual users, rather than groups of users.

More recently, it was shown in [10-12] a group composite alternatives recommender (GCAR) framework that addresses the outlined limitations, and provides a diverse set of group package recommendations based on multi-criteria decision optimization. However, the framework is a generic one and has not been applied on a realistic complex problem, like the microgrid renewable energy sources investment.

\section{Overview of PMOIR and Contribution to Practice}

Consider an example of a power microgrid of a university campus, such as George Mason University (GMU) Fairfax VA campus. As depicted in Figure 1, such microgrid involves a number of interrelated power components, such as services, including lighting, cooling and water heating; utility contracts, such as GMU contract with Dominion Virginia Power; and backup generators. Suppose that a group of decision makers are planning to invest in the renewable energy components of this microgrid, namely the solar photovoltaic cells and wind turbines.

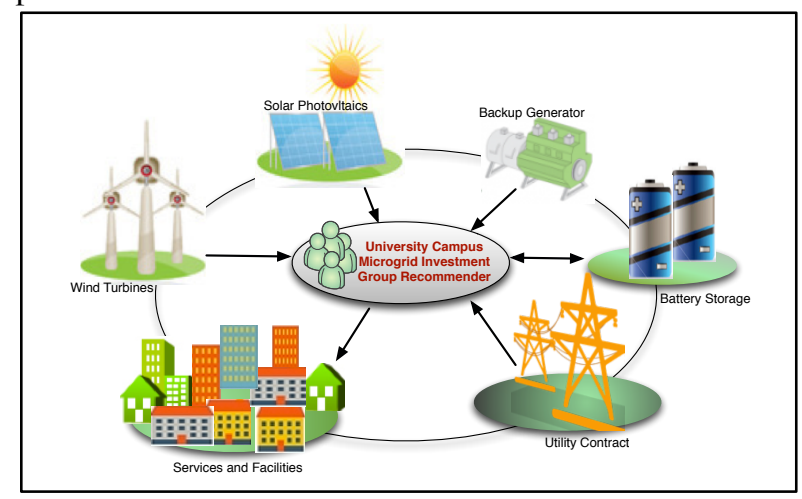

Figure 1. Example of a university campus microgrid

Investing in these components is not trivial process because their power supply is unpredictable and may drop suddenly, which will require either immediately supplementing it from other power components like batteries or reducing the demand. Thus the components interact with each other as a package: whenever the supply from the renewable components drops, other components may compensate for it. In addition, the microgrid system needs to decide on an hourly basis how much power is to be supplied or used by each component.

This microgrid consists of both the installed energy components and others that may have to be purchased later in the time horizon to meet the entire campus's future power demands. The energy managers have recently seen a significant growth in power demand, and because the campus is continuing its expansion rapidly, they realize that the existing energy components will not be able to satisfy future power demand.

For these reasons, a group of decision makers from different departments have to determine the best investment and operation recommendations to satisfy current and future power demand while also addressing the optimal operation of the new components with the installed ones. The energy operation and investment recommendations include optimal settings and values for decision control variables, such as: whether a new component should be purchased or not; and the amount of power generated from the resource components (e.g., backup generators, and renewable resources) or consumed by service components (e.g., heating, air conditioning, and lighting) in each time interval. The goal is to find a small set of optimal operational and investment recommendations that achieves the right balance of the three criteria (NPV, GHG emissions, and demand satisfaction ratio) while satisfying the group of decision makers, who have different views on appropriate weights for the criteria.

The motivation of the PMOIR work stems from a range of studies, performed by our team at GMU, for operational and planning recommendations in related real-world engagements. These studies include (1) optimization modeling of the microgrid power components to advise GMU energy management team on optimal extension of cooling, heating capacity using a variety of options including co-generation [14]; (2) a study to recommend optimal setting of peak-demand to be enforced by GMU energy management system [13]; and (3) a study for Dominion Virginia Power on integration of power storage capacity in conjunction with renewable sources of energy in their networks, based on power network modeling and optimization [8]. The work on PMOIR reported in this paper builds on our prior experience and attempts to build a more general framework to enable a systematic process of decision making on renewable energy investment for a group of decision makers and stake-holders. 


\section{Overview of the GCAR Framework}

In this section, we first describe the recommendation space of GCAR framework proposed in [11], then, we explain the recommendation process implemented by this framework and the intuition behind this process.

Recommendation space $\mathrm{R}$, include the settings and values for decision control variables of interrelated power sources; each recommendation alternative $a \in R$ is mapped to a utility vector $\overrightarrow{\mathrm{u}}=\left(u_{1} \ldots, u_{n}\right)$ from an $n$ dimensional utility space, such that: $\forall_{i}, 1 \leq i \leq n$, $u_{i}: R \rightarrow[0,1]$. The components of a utility vector $\vec{u}=$ $\left(u_{1}, u_{2}, \cdots, u_{n}\right)$, are associated with criteria such as the Net Present Value NPV, GHG emissions, etc., which are previously defined. Each criterion has an associated domain $\mathrm{D}_{\mathrm{i}}, 1 \leq i \leq n$, and each domain $\mathrm{D}_{\mathrm{i}}$ has a total ordering "better than" denoted $\geqslant_{D i}$. For example, for domain NPV, $a_{1} \geqslant_{N P V} a_{2} \Leftrightarrow a_{1} \geq a_{2}$.

For a given group of $m$ users, the utility of each user $j$, denoted by: $\forall_{j}, 1 \leq j \leq m, \mathrm{U}_{J}:[0,1]^{n} \rightarrow[0,1]$, maps a vector of criteria $u_{1} \ldots, u_{n} \in[0,1]$ into a user utility $\mathrm{U}_{j}\left(u_{1} \ldots, u_{n}\right) \in[0,1]$, and the group utility is denoted by: $\mathrm{U}:[0,1]^{n} \rightarrow[0,1]$.

$\mathrm{U}_{j}$ and $\mathrm{U}$ define a utility associated with each alternative $a \in R$. Therefore, the user recommendation alternative utility for recommendation $a$ is defined by: $R U_{j}: R \rightarrow[0,1]$, where $R U_{j}(a)=\mathrm{U}_{j}\left(u_{l}(a), \ldots, u_{n}(a)\right)$, and the group recommendation alternative utility is defined by: $R U: R \rightarrow[0,1]$, where $R U(a)=\mathrm{U}\left(u_{l}(a), \ldots, u_{n}(a)\right)$.

The recommendation process implemented by this framework is depicted in Figure 2.

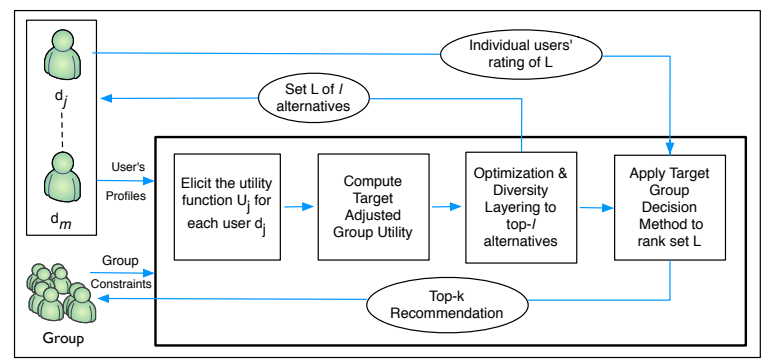

\section{Figure 2. Group Composite Alternatives Recommender (GCAR) framework}

As shown in the diagram, the process starts when a group of users submits a request to the group recommender. This request specifies the group's decision constraints on recommendation alternatives.

To generate the top-k recommendations, the recommender follows six steps: (1) Elicit the utility function for each user; (2) Estimate the group utility function; (3) use this to find an optimal recommendation alternative; (4) Use diversity layering to generate a diverse set of $l$ recommendations that contains the optimal recommendation; (5) Rank or rate (depending on the method used in the last step) this set by each user; (6) apply a voting method to refine the final $k$ recommendations.

The intuition behind this process is as follows: First, the framework applies alternative voting methods to make the final recommendations for a group of decision makers. Different voting methods are used by different people, and the choice of method usually depends on the domain, the group's characteristics, and what property people want to satisfy. There is no single method considered to be generally superior to all others and fully achieves fairness [2].

This framework considers six voting methods, any one of which can be used to instantiate the framework. Three are based on well-known and commonly used aggregation strategies: average, least misery, and average without misery strategies. Two are voting methods based on individuals' rankings: instant runoff voting (IRV) and the hybrid Condorcet-IRV method. The last one is a method called: the structurally adjusted average method, which has been developed in [11] to take into account the influence of decision makers within the group and the dissimilarity of opinions among them. (Applying alternative voting methods is the last step of the process in Figure 2). Work [11] showed that all are possible to apply in the framework, but our choice in this paper is the Average method.

Voting methods can be applied only when there are a small number of alternatives; In PMOIR, however, we have different interactive power components, each has many possible models and capacities. Also, there are many possible combinations of these components, which make the search space exponentially in number of components or even infinite, so that it is impractical to apply a voting method directly on such a space. We first need to restrict the large search space to a small, highly relevant set that can then be refined through voting.

To carry out this reduction, mathematical optimization is applied to produce a small set of recommendations that are close to optimal, and sufficiently diverse that group members will have enough flexibility. The key idea is to create a subset of divers recommendations that correspond to different individuals' utility functions, while preserving a bounded distance from the optimal group utility score in order to provide the right balance between optimality and diversity, see Figure 3. The recommendation space is partitioned into $q$ layers starting from the layer that includes the optimal recommendation, which maximizes the group utility $U$. The second layer includes the recommendations that 
are close to the optimal recommendation having a total utility value no less than the maximum group utility minus $\varepsilon$, where $\varepsilon$ corresponds to a percentage of the maximum group utility score. The third layer includes the recommendations indicating a total utility value no less than the maximum group utility minus $2 \varepsilon$.

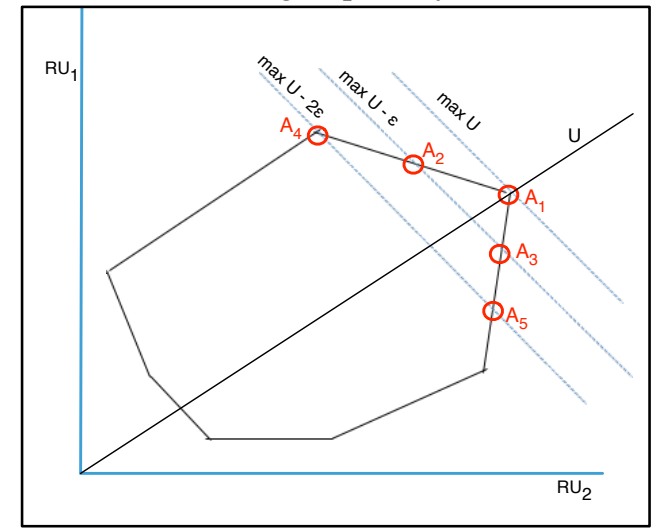

Figure 3. Diversity layering

Generally, Recommendations in the $i$-th layer have a utility value no less than the maximum group utility function minus $(i-1) \varepsilon$. Within each layer, we select $n$ recommendations to maximize each dimension of the recommendation space in turn. This explains the second-last step in Figure 2 (Optimization and Diversity Layering).

However, to complete the optimization and diversification, we need to estimate the group utility function that captures the whole group's preferences, this explains the second step in Figure 1. This group utility function is parameterized on the basis of the target group decision-making method, and it must be based on the utility functions of the individual users, which are not known to the system and need to be extracted from the individuals. This is the first step in Figure 1. For more details of these steps, refer to work [11].

\section{Optimization Formalization}

Assume that a microgrid consists of a set of power components $C=\left\{c_{1}, \ldots, c_{k}\right\}$ that includes a subset of components available at the initial time horizon, denoted by (initAvaC), and a subset of components that are not yet available but might be purchased later in the time horizon, denoted by (notInitAvaC), where $C=$ initAvaC $\mathrm{U}$ notInitAvaC. These components can be considered power-producing resources, such as backup generators and solar panels, or power-consuming services, such as lighting and heating.

The time horizon $T$ is a set of discrete hourly time intervals, $T=\{1, \ldots, N\}$ where $N=24$ if the time horizon is one operational day, and $N=8760$ if the time horizon is one operational year with an hour long interval length, and so on. An interval length of 1 means that each time interval is an hour long.

Generally, every component $i \in C$ is associated with the following:

1. A vector of controls $\overrightarrow{a_{\imath}}=\left(a_{i 1} \ldots, a_{i N}\right)$, which represents the control actions that component $i$ takes over time horizon $T$, and where each $a_{i t}, 1 \leq i \leq k$, $1 \leq t \leq N$, is the control action that component $i$ takes at time $t$. Therefore, the control actions for all components over the time horizon $T$ is represented as matrix $A$ :

$$
A=\left(\begin{array}{c}
\overrightarrow{a_{1}} \\
\vdots \\
\overrightarrow{a_{k}}
\end{array}\right)=\left(\begin{array}{ccc}
a_{11} & \cdots & a_{1 N} \\
\vdots & \ddots & \vdots \\
a_{k 1} & \cdots & a_{k N}
\end{array}\right)
$$

These control actions are defined as follows:

- avail $_{i}$ indicates whether component $i$ is available at the initial time interval; $\forall i \in C$, avail $_{i}=1$ if component $i$ is available and 0 otherwise.

- buyFlag $_{i}$ indicates whether new component $i$ should be purchased, where $\forall i \in$ notInitAvaC, buyFlag $_{i}=1$ if component $i$ is to be purchased and 0 otherwise. In addition, $\forall i \in$ initAvaC, buyFlag $_{i}=0$.

- on $_{i, t}$ indicates whether component $i$ supplies or uses an amount of power at time interval $t$, such that $\forall i \in C$, on $_{i, t}=1$ if $i$ supplied or used power at $t$ and 0 otherwise.

- $k w_{i, t} \in \mathbb{R}$ indicates how much power should be supplied by each component $i \in C$ in each time interval $t \in T$.

2. A number of metrics, such as cost, GHG emissions, and number of payments during the time horizon, where each payment consists of a specific amount paid at a specific time interval. These metrics are discussed for each component type in the following subsections.

3. A number of operational and investment constraints, in terms of control actions $\overrightarrow{a_{l}}$. For example, the capacity in $k w$ of a power generator is a constraint on the amount of power that this component can generate.

Every operation and investment decision option for this problem will involves the values of all the component decision control vectors over the time horizon. In addition, each option is associated with a utility vector $\vec{u}=\left(u_{1} \ldots, u_{n}\right)$ from an $n$-dimensional utility space, such that $\forall_{i}, 1 \leq i \leq n, u_{i}: R \rightarrow[0,1]$, where each utility $u_{i}$ has a specific domain $D_{i}$ and represents a specific criterion. For example, $u_{1}$ represents the NPV, $u_{2}$ represents the GHG emissions, and $u_{3}$ represents the demand-satisfaction ratio, where 
$\sum_{i=1}^{n} u_{i}=1$. Furthermore, each utility has a global weight, utilityWeight ${ }_{i}$ such that $\sum_{i=1}^{n}$ utilityWeight $_{i}=1$. Therefore, the total utility of all components, given their control action matrix $A$, denoted by TotalUtility : $R \rightarrow[0,1]$, is defined as

TotalUtility $(A)=\sum_{i=1}^{n}$ utilityWeight $_{i} \times$ utility $_{i}$

The overall optimization problem is to maximize the total utility over the action matrix $A$, subject to three kinds of constraints: the generic balance constraints for the entire problem model, the utilitydefinition constraints for the entire model, in terms of the component metrics, and the specific constraints for each power component. Formally, the optimal control action matrix $A^{o}$ is the one that maximizes the utility the microgrid system can achieve:

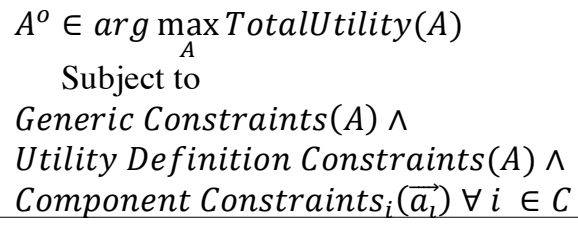

In the following subsections, we describe in details each of the first two constraints, leaving the power component constraints for the next section.

\subsection{Generic Constraints}

In addition to the specific constraints for each component $i$ given its control actions vector, $\left(\overrightarrow{a_{l}}\right) \forall i \in C$, there are generic constraints for the entire model, given the control action matrix $A$, that need to be satisfied. These are as follows:

- For a stable power supply to be maintained, the sum of power supply and power demand for any time interval must equal 0 ; that is,

$\forall t \in T \sum_{i=1}^{k}$ power $_{i}\left(a_{i t}\right)=0$

where power $_{i}$ is the power in $\mathrm{kW}$ that component $i$ produces or consumes, given the control actions vector $\overrightarrow{a_{\imath}}$, in any time interval $t$. This value is positive if the component supplies power, negative if it receives power, and zero if it is unavailable at $t$ or is turned $O F F$.

- In any time interval, if a component $i$ is $O F F$, the output or input power from or to $i$ for this time interval must equal 0 ; that is,

$$
o n_{i, t}=0 \Rightarrow k w_{i, t}=0 \quad \forall i, t
$$

- For each component, if any amount of power is generated or used by the component in a time interval, the component must be $O N$ for this time interval:

$$
k w_{i, t} \geq m \Rightarrow o n_{i, t}=1 \quad \forall i, t
$$

where $m \in \mathbb{R}$, and $m>0$.

- For each component, at any time interval, the value of control action on $_{i, t}$ cannot exceed the value of control action avail $_{i}$ :

$$
\text { on }_{i, t} \leq \text { avail }_{i} \quad \forall i, t
$$

- At any time interval, the amount of power produced or consumed by any component must be bounded by a minimum and a maximum value:

$$
-M \times o n_{i, t} \leq k w_{i, t} \leq M \times o n_{i, t} \quad \forall i, t
$$

where $M$ is a constant.

- For each initially available component, the value of the control action buyFlag $i$ is 0 and that of avail $_{i}$ is 1 :

buyFlag $_{i}=0$, avail $_{i}=1 \quad \forall(i \in$ initAvaC $)$

- For each component that is not initially available, the value of the control action avail $i$ is equal to the value of the control action buyFlag ${ }_{i}$ :

$$
\text { avail }_{i}=\text { buyFlag }_{i} \quad \forall(i \in \text { notInitAvaC })
$$

- The demand-satisfaction ratio, which is explained in the next subsection, must be greater than or equal to an accepted value. For example, the microgrid energy system must satisfy at least $95 \%$ of the total power demand:

$$
\text { demSatRatio }_{A} \geq \text { accepted Value }
$$

\subsection{Utility Definition Constraints}

In this subsection, we describe the global utility definition constraints for the entire model in terms of the component metrics. The global utility is the additive combination of the net present value (NPV), the GHG emission, and the demand-satisfaction ratio for the entire model.

Net present value (NPV) utility. NPV is defined as

$$
N P V=\sum_{t=1}^{N} \frac{\text { payPerInt }}{(1+r)^{t-1}}
$$

where $r$ is the discount rate per interval $t \in T$, and payPerInt $t$ is the total amount of payments for each cost category of each component $i$ at interval $t$, which is calculated for each component type as described in Section 6.

GHG emissions utility. The total GHG emissions, denoted by totalCo2Value, is the sum of the emissions generated by all components. Formally,

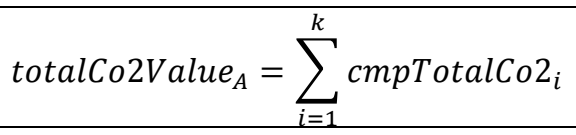

where $\mathrm{cmpTotalCo} 2_{i}$, for each component type, is calculated as described in Section 6. 
Demand-satisfaction ratio utility. The demandsatisfaction ratio, denoted by demSatRatio, is the ratio of the total supplied power to the total needed power:

demSatRatio $_{A}=$ totalSupK $_{A} \div$ totalDemKW

\section{Power Component Modeling}

To support optimization, we mathematically modeled different power components based on the models described in work [1].

\subsection{Utility Power Contract}

A utility contract is a service agreement between a utility company and its business partners. It defines all the commercial terms for the sale of power between the two parties, including the cost of using the power, the cost of the maximum peak demand bound, and the penalty charge for exceeding this bound.

Cost: Each contract component $i$ has only one cost category (conCost) for each time interval. This typically includes both the peak demand charge and the total power consumption charge. The peak demand charge, denoted by peakDem, measures the maximum rate of power consumption for any time interval. The total power consumption charge, denoted by powerCons, measures the rate of power consumption in the specific billing period. Formally, $\forall i \in$ Contracts, such that Contracts $\subset C$, and $\forall t \in T$, conCost $_{i, t}$ is defined as

conCost $_{i, t}=$ powerCons $_{i, t}+$ peakDem $_{i, t}$, where powerCons $_{i, t}=k w_{i, t} \times$ powerPricePerKw $\left(k w_{i, t}\right)$, and peakDem ${ }_{i, t}=k w_{i, t} \times$ peakPricePerKw $\left(k w_{i, t}\right)$

The powerPricePerKw and the peakPricePerKw are functions that are represented as piece-wise or stepwise linear functions.

Payments: This cost category (conCost) has number of payments (noPay) during the time horizon, and each payment $p$ has an amount (payAmt) that is paid at a specific time interval (payInt). For example, if this cost category is paid monthly, there will be 12 payments in a one-year time horizon. On an hourlybasis time horizon, the first payment $(p=1)$ will be made at $t=730$, the second $(p=2)$ at $t=1460$, and so on. Formally,

$$
\begin{aligned}
& \forall(i \in \text { Contracts, } t \in T, p \in\{1, \ldots, \text { noPay }\}): \\
& \text { payAmt }_{i, p}=\sum_{t} \text { conCost }_{i, t} \text {, such that: } \\
& \left.t \in \text { payInt }_{p-1}+1, \ldots, \text { payInt }_{p}\right\}
\end{aligned}
$$

For example, at the second month, this payment amount will be calculated from $t=731$ to $t=1460$.
GHG emissions: The total GHG emission of each contract component $i$, denoted by conTotalCO2 $2_{i}$ is the sum of conCO2, which is the GHG emission of component $i$ in each time interval. Formally, $\forall i \in$ Contracts, conTotalCO $2_{i}$ is defined as

$\operatorname{conTotalCO}_{i}=\sum_{t=1}^{N} \operatorname{conCO}_{i, t}$, where
$\operatorname{conCO}_{i, t}=k w_{i, t} \times{\operatorname{conCO} 2 p e r K w_{i}}^{*}$

and conCO2perKw $w_{i}$ is the GHG emission (in Btu) produced in the consumption of each kilowatt of power.

Operational and investment constraints: At any time interval, the total $k w$ consumption must not exceed the contract peak demand bound:

$k w_{i, t} \leq$ peakDem $_{i} \quad \forall i, t$

\subsection{Backup Power Generator}

A generator requires fuel to operate and typically has an efficiency function of fuel consumption $\left(E f f i_{i}\right)$ based on the amount of power it generates. This function, which can be defined as a piece-wise linear function or a step-wise linear function, determines the amount of fuel needed for each kilowatt generated.

Cost: Every backup generator has three cost categories: fuel, maintenance, and depreciation -the difference between its present value and its residual value at the end of the time horizon. There is also a fourth cost category for each new generator purchased during the time horizon, which is equal its price. Formally, $\forall i \in$ Generators, such that Generators $\subset$ $C$, and $\forall t \in T$, the fuel and depreciation cost categories are defined as:

FuelCost $_{i, t}=$ fuelPrice $_{i, t} \times E_{\text {ffi }}\left({ }_{i}\left(k_{i, t}\right) \times k w_{i, t}\right.$
DeprCost $_{i}=$ PresenValue $_{i}-$ ResidualValue $_{i}$

Payments: While the FuelCost could be paid monthly, with 12 payments in a time horizon of one year, the MaintCost is paid annually and the DeprCost is considered only at the end of the time horizon; that is, when $t=N$, Formally,

$\forall(i \in$ Generators $, t \in T, p \in\{1, \ldots$, noPay $\})$ :

Maintenance's payAmt $_{i, p}=$ MaintCost $_{i} \times$ avail $_{i}$

Depreciation's payAmt $t_{i, p}=$ DeprCost $_{i} \times$ avail $_{i}$

Equipment's payAmt $_{i, p}=$ EquipCost $_{i} \times$ buyFlag $_{i}$

The payment amount for the fuel cost category is calculated similarly to the calculation for the contract cost in the previous subsection.

GHG emission: The total GHG emission of a generator component is calculated as for a contract component. 
Operational and investment constraints: For any generator component, at any time interval, the output power must not exceed the generator's capacity:

$k w_{i, t} \leq$ genCapacity $_{i} \forall i, t$

\subsection{Renewable Energy Resources}

Renewable energy systems (e.g., photovoltaic systems, and wind turbines) generally supply energy that comes from natural sources, such as sunlight and wind. While these are much more environmentally friendly than non-renewable energy resources, they are much more expensive to use and depend on environmental factors like sunshine or wind activity, which makes it difficult to control their output power. The output of these components is represented as the predicted power (in $\mathrm{kW}$ ) generated over a time horizon, denoted by predictedOutput $t_{i, t} \quad \forall(i \in$ Renewables, $t \in T)$, such that Renewables $\subset C$.

Cost and Payments: Each renewable resource has two main cost categories: annual maintenance and depreciation. As with the generators, there is also a cost of new equipment for resources newly purchased during the time horizon. These categories and their payment amounts are defined and calculated like the generator cost categories.

GHG emission: Because renewable resources do not typically use fuel to produce power, the total GHG emission of each renewable component is equal to 0 .

Operational and investment constraints: In any time interval, the power used from any renewable resource component cannot exceed the power generated by it:

$$
k w_{i, t} \leq \text { predictedOutput }_{i, t} \quad \forall i, t
$$

\subsection{Battery Storage Units}

A battery storage component is in one of three performance states at a given time: discharging (supplying power), charging (consuming power), or idle. A battery typically has a limited number of design charge-discharge cycles, denoted by batLifeCyc, before it is considered inefficient and needs to be replaced.

Cost and Payments: Like the renewable components, a battery has two main cost categories, maintenance and depreciation, and a third category for new battery purchases. The depreciation cost is the cost of wear caused by using the battery. To determine the cumulative number of cycles used, we divide the value of the used cumulative charge/discharge cycles of the battery, cumCharDischar, by the power in kilowatts for a single cycle life energy, cycEnergy. This result, denoted by cumCyc, is multiplied by the new battery cost and divided by batLifeCyc. Formally, $\forall i \in$ Batteries, such that Batteries $\subset C$, and $\forall t \in T$, the depreciation cost is defined as:

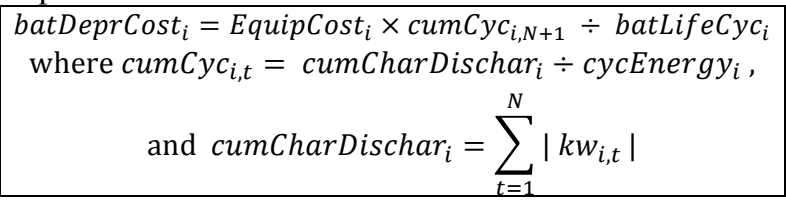

All payments amounts are calculated as for the generators.

GHG emission: As with the renewable resources, the total GHG emission of each battery component is equal to 0 .

Operational and investment constraints: For any battery component, at any time interval, $\forall(i \in$ Batteries, $t \in T$ ),

- The discharge power amount must equal at least the minimum discharge rate:

$$
k w_{i, t} \geq \text { minDischargeRate }_{i}
$$

- The charging power amount must not exceed the maximum charge rate:

$$
k w_{i, t} \leq \text { maxChargeRate }_{i}
$$

- At the beginning of the time horizon, when $t=1$, the discharge power amount cannot exceed the battery's initial energy:

$$
k w_{i, t} \leq \text { batInitialEnergy }_{i}
$$

- For the remaining time intervals, where $t \in\{2, \ldots, N\}$, the discharge power amount cannot exceed the battery's current charge. That is,

$k w_{i, t} \leq$ CurrentCharge $_{i, t-1} \quad$, where
CurrentCharge $_{i, t+1}=$ CurrentCharge $_{i, t}-k w_{i, t}$

- The battery's current charge must not exceed its capacity:

\begin{tabular}{l} 
CurrentCharge $e_{i, t} \leq$ batCapacity $_{i}$ \\
- At the beginning of the time horizon, when $t=1$, the \\
cumulative charge-discharge power is equal to zero: \\
\hline cumCharDischar ${ }_{i, t=1}=0$ \\
\hline
\end{tabular}

\subsection{Power-Consuming Services}

Power-consuming components are services that contribute to the power demand, such as HVAC, lighting, and water heating. Each service component $i$, requires an amount of power, denoted by serPredictedDemand, to run during each time interval.

Because there are no power-related costs to operating a service other than the cost of supplying power, the cost of any service component for any time interval is 0 . Therefore, there are no payments for these components. In addition, their total GHG emission is equal to 0 . 
Operational and Investment Constraints: In any time interval, the power supplied to service component $i$ must be equal to the power needed if the service is $O N$ and 0 otherwise:

$k w_{i, t}=$ serPredictedDemand $_{i, t} \times$ on $_{i, t} \quad \forall i, t$

\section{Experimental Study}

We implement the power optimization model for PMOIR as a mixed-integer linear programming model using Optimization Programming Language (OPL) and CPLEX Studio to decide on the power resource investment and operation. The question we address in this experiment is whether PMOIR is practical for generating a small set of optimal and diverse recommendations within a reasonable amount of time. To answer this question, we implement PMOIR using the GCAR framework.

In this study, the utility function of each member of a group of three decision makers is generated synthetically. The group utility function is estimated using the average group decision-making method. The study considers three different microgrid sizes: a small microgrid of up to 50 power components, a mediumsized microgrid of up to 100 components, and a large microgrid of up to 200 components. All the power components belong to the types described and modeled in this paper. In addition, all three microgrids are operated over three different time horizons: one year, three years, and five years.

The data sets are generated with real data from [15], which provides annual energy usage information (in $\mathrm{kWh}$ ) from 1989 to 2010 at the University of Texas at Austin. Ten data sets are generated and tested for each microgrid over each time horizon. The testing is performed on 2.6 workstation with Intel Core i7 processor and a memory of $16 \mathrm{~GB} 1600 \mathrm{MHz}$ DDR. The mean resolution times for all three microgrid sizes over the three time horizons are depicted in Figure 4.

The largest data set in this study, which involves 200 components over a five-year time horizon, contains over 23 million constraints, and about 18 million variables, of which over 8 million variables are binary and nearly 10 million variables are continuous. This largest dataset is solved in less than five hours of solver time, meaning PMOIR framework is feasible for use with medium-sized and large microgrids to generate a small set of optimal and divers recommendations within a reasonable time for investing millions of dollars. Note that the resolution time includes the time required for the group utility optimization and the optimization over each decision maker's utility to diversify the recommendation set.

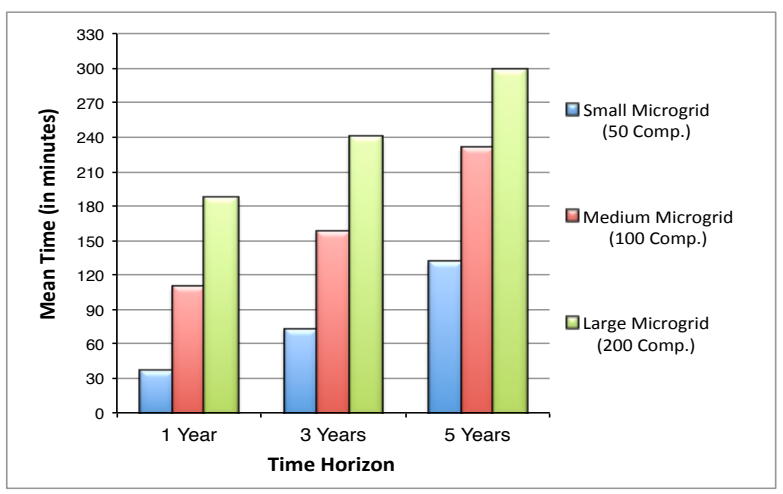

Figure 4. Experimental mean resolution time for three microgrid sizes over three time horizons

For the statistical analysis, we calculate the Confidence Interval at a 95\% level for the estimated mean of the time resolution for each microgrid size and over each time horizon. The results are illustrated in Table 1.

Table 1. Confidence interval at level $95 \%$ for the estimated mean of the time resolution (in minutes)

\begin{tabular}{|c|c|c|c|}
\hline \multirow{2}{*}{ Microgrid Size } & \multicolumn{3}{|c|}{ Time Horizon } \\
\cline { 2 - 4 } & 1 year & 3 years & 5 years \\
\hline Small & $36.49 \pm$ & $73.09 \pm$ & $130.38 \pm$ \\
(50 Components) & 1.22 & 2.17 & 2.13 \\
\hline Medium & $109.06 \pm$ & $158.15 \pm$ & $230.21 \pm$ \\
(100 Components) & 1.47 & 2.34 & 3.28 \\
\hline Large & $187.06 \pm$ & $239.36 \pm$ & $299.02 \pm$ \\
(200 Components) & 2.12 & 3.36 & 4.22 \\
\hline
\end{tabular}

As shown in this table, the mean resolution time for the smallest data set (50 components over a one-year time horizon) is 36.49 minutes, with upper and lower bounds of 1.22 minutes. The mean resolution time for the largest data set (200 components over a five-year time horizon) is 299.02 minutes, with upper and lower bounds of 4.22 minutes.

After the diverse set of recommendations is generated, they are to be presented to each decision maker in descending order of group utility, and each decision maker ranks the set in accordance with his or her preferences. Finally, the target voting method is applied on the ranked set of recommendations to determine the final top $k$ recommendations.

\section{Conclusions and Future Work}

In this paper, we developed a Power Microgrid Operation and Investment Recommender (PMOIR) to 
recommends a set of optimal, or near optimal, operational and investment decisions in a power microgrid to a group of decision makers who need to maximize the global utility of the microgrid while taking into account all the component's interactions, and multiple potentially conflicting views on the importance of various criteria. In addition, we modeled the different components, formalized the optimization problem, and implemented the power optimization model as a MILP model using OPL and CPLEX Studio. Finally, we validated PMOIR framework with an experimental study demonstrating its feasibility, in terms of computational time, to be applied on microgrids involving 200 power components, over a five-year time horizon, with around 8 million binary variables.

Further exploration is possible in many areas, for example, future research can model a range of power and renewable energy components going beyond the five formalized and modeled in this work. In addition, it would be worth addressing the possibility of leveraging the existing energy market to sell excess capacity during time intervals with low demands. Also, we can simplify the investment model by considering a monthly basis time horizon instead of an hourly basis, by giving probabilities of the peak demand each month. Thus, this will make the decision process much easier and with shorter resolution time.

\section{References}

[1] Altaleb, H., Market-Based Decision Guidance Framework for Power and Alternative Energy Collaboration, George Mason University - WRLC, 2015.

[2] Arrow, K., "A Difficulty in the Concept of Social Welfare", Journal of Political Economics, 58(1950), pp. 328346.

[3] Baringo, L., and Conejo, A.J., "Strategic Wind Power Investment", IEEE Transactions on Power Systems, 29(3), 2014,pp. 1250-1260.

[4] Brodsky, A., Henshaw, S.M., and Whittle, J., "Card: A Decision-Guidance Framework and Application for Recommending Composite Alternatives", Proceedings of the 2008 ACM conference on Recommender systems, 2008, pp. 171-178.

[5] Fazlollahi, S., Mandel, P., Becker, G., and Maréchal, F., "Methods for Multi-Objective Investment and Operating Optimization of Complex Energy Systems", Energy, 45(1), 2012, pp. 12-22.

[6] Interdonato, R., Romeo, S., Tagarelli, A., and Karypis, G., "A Versatile Graph-Based Approach to Package Recommendation", 2013,
[7] Lakiotaki, K., Tsafarakis, S., and Matsatsinis, N., "UtaRec: A Recommender System Based on Multiple Criteria Analysis", Proceedings of the 2008 ACM conference on Recommender systems, 2008, pp. 219-226.

[8] Levy, R., Brodsky, A., and Luo, J., "Decision Guidance Approach to Power Network Analysis and Optimization", In Proceedings of the 18th International Conference on Enterprise Information Systems, 2016, pp. 109-117.

[9] Masthoff, J., "Group Recommender Systems: Combining Individual Models". Recommender Systems Handbook, Springer US, 2011, pp. 677-702.

[10] Mengash, H., and Brodsky, A., "GCAR: A Group Composite Alternatives Recommender Based on MultiCriteria Optimization and Voting", in System Science (HICSS), 47 ${ }^{\text {th }}$ Hawaii Internatioal Conference, 2014, pp. $1113-1121$.

[11] Mengash, H., and Brodsky, A., "A Group Package Recommender Based on Learning Group Preferences, MultiCriteria Decision Analysis, and Voting", EURO Journal on Decision Processes, Springer Berlin Heidelberg, 2015, pp. 275 - 304.

[12] Mengash, H., and Brodsky, A., "Tailoring Group Package Recommendations to Large Heterogeneous Groups Based on Multi-Criteria Optimization", in System Science (HICSS), 47 ${ }^{\text {th }}$ Hawaii Internatioal Conference, 2016, pp. 1537 - 1546 .

[13] Ngan, C.K., and Brodsky, A., "DGLS System: Decision Guidance for Optimal Load Shedding in Electric Power Microgrids", in Proceedings of the International Conference on Artificial Intelligence (ICAI), Las Vegas, USA, 2013

[14] Ngan, C.K., Brodsky, A., Egge, N., and Backus, E., "Optimizing Power, Heating, and Cooling Capacity on a Decision-Guided Energy Investment Framework", in Enterprise Information Systems: $15 \mathrm{~h}$ International Conference, 2014, pp. 154-173.

[15] http://en.openei.org/datasets/dataset/commercial-andresidential-hourly-load-profiles-for-all-tmy3-locations-inthe-united-states/resource/b341f6c6-ab5a-4976-bd07adc68a2239c4.2015.

[16] Shi, Y., Larson, M., and Hanjalic, A., "Collaborative Filtering Beyond the User-Item Matrix: A Survey of the State of the Art and Future Challenges", ACM Comput. Surv., 47(1), 2014, pp. 1-45.

[17] Wang, H., and Huang, J., "Hybrid Renewable Energy Investment in Microgrid", in Smart Grid Communications (SmartGridComm), 2014 IEEE International Conference, 2014, pp. 602-607.

[18] Wang, H., Huang, J., Lin, X., and Mohsenian-Rad, H., "Exploring Smart Grid and Data Center Interactions for Electric Power Load Balancing", SIGMETRICS Perform. Eval. Rev., 41(3), 2014, pp. 89-94.

[19] Xu, L., Ruan, X., Mao, C., Zhang, B., and Luo, Y., "An Improved Optimal Sizing Method for Wind-Solar-Battery Hybrid Power System", IEEE Transactions on Sustainable Energy, 4(3), 2013, pp. 774-785. 